\title{
Avances en la investigación del virus dengue en Colombia: papel de los microARNs celulares en la respuesta anti-dengue virus
}

\author{
Juan Camilo Castrillón-Betancur y Silvio Urcuqui-Inchima
}

\section{Advances in dengue virus research in Colombia: the role of cellular microRNAs as an anti-dengue virus response}

Dengue is one of the most important mosquito-borne diseases, and its incidence has increased at an alarming rate in recent years, becoming a real public health problem. Currently, there is no vaccine or medication or proper treatment for dengue control. Considering this situation, it is necessary to prioritize the search for new alternatives and strategies for dengue prevention and control, in order to reduce not only the economic burden of endemic countries, but also to improve the quality of life of patients. In this regard, a brief reflection on some aspects related to the search for new alternatives in Colombia is presented. This is focused on the use of microRNAs, which could be a new strategy with great therapeutic potential.

Key words: Dengue, microRNAs, anti-dengue virus response.

Palabras clave: Dengue, microARNs, respuesta anti-dengue.

$\mathrm{E}$ 1 dengue es una de las enfermedades más importantes transmitidas por la picadura de mosquitos del género Aedes y su incidencia ha aumentado a un ritmo alarmante en los últimos años, al punto que se ha convertido en un verdadero problema de salud pública ${ }^{1}$. La situación se ha complicado aún más, ya que el vector se ha extendido rápidamente a otras regiones que están más allá del área tropical o subtropical. Esto como consecuencia al cambio inesperado de algunas variables ambientales como la precipitación y la temperatura; $\mathrm{y}$ de cambios sociales como el crecimiento demográfico y la rápida urbanización no planificada, junto con el deterioro de programas para la vigilancia, prevención y control del mosquito ${ }^{2}$.

El principal vector implicado en la transmisión del dengue virus (DENV) es Aedes aegypti, que vive en hábitats urbanos y recipientes artificiales, y en menor medida Aedes albopictus, un vector inicialmente descrito en Asia, pero más recientemente se ha extendido a las Américas, incluyendo Colombia y Europa, debido en gran medida, al comercio internacional ${ }^{3,4}$. Los seres humanos infectados son los principales portadores y multiplicadores del virus y se convierten en fuente del virus para mosquitos no infectados.

El dengue tiene un amplio espectro de presentaciones clínicas, a menudo con evolución clínica y resultados impredecibles. Aunque la mayoría de los pacientes se recupera después de un curso clínico benigno y de resolución espontánea (fiebre alta acompañada de dos de los siguientes síntomas: cefalea intensa, dolores musculares y articulares, náuseas, vómito, inflamación de los ganglios o erupción cutánea), una pequeña proporción progresa a una enfermedad grave, caracterizada principalmente por aumento de la permeabilidad vascular, con hemorragia o sin ella y compromiso de órganos. Las próximas $24-48$ h de la etapa crítica pueden llegar a ser letales, por lo que se necesita de atención médica adecuada para evitar complicaciones y el riesgo de muerte. La rehidratación intravenosa es el tratamiento de elección ya que puede reducir la tasa de letalidad a menos de $1 \%$ en los casos graves $^{5}$. Sin embargo, evitar que el dengue progrese a las condiciones clínicas más graves, muchas veces es difícil ya que entre los aspectos encontrados como críticos en la atención al paciente está la falta de una consulta oportuna, fallas en el diagnóstico y las barreras de acceso a los servicios de salud, principalmente en zonas rurales. Igualmente se debe tener presente que actualmente no existe ni vacuna ni un medicamento o tratamiento adecuado para el control del dengue.

En la actualidad, el único método para controlar o prevenir la transmisión del DENV es a través del control de los mosquitos vectores, que puede ser mediante la prevención del acceso de los mosquitos a sus hábitats de desove, eliminando los recipientes de almacenamiento de agua domésticos. Igualmente, aplicando insecticidas apropiados u orientando a las personas para que adquieran la costumbre de usar por ejemplo mosquiteros en ventanas y ropa de manga larga ${ }^{6}$. Sin embargo, el
Universidad de Antioquia Facultad de Medicina, Grupo Inmunovirología, Medellín, Colombia.

Los autores manifiestan que no existe, de manera directa o indirecta, conflicto de intereses alguno.

Financiamiento: Universidad de Antioquia, UdeA, CODI (mediana cuantía) acta 624.

Recibido: 8 de noviembre de 2016

Aceptado: 27 de enero de 2017

Correspondencia a: Silvio Urcuqui-Inchima silviourcuqui@gmail.com 
apoyo técnico y la orientación periódica de este tipo de programas para la prevención y el control del vector y por ende, del dengue, demandan un aumento de recurso humano y en consecuencia, grandes recursos económicos. Además, la presión en el sistema de salud es significativa debido a que la carga económica para el dengue supera a la de otras enfermedades virales. El costo de la enfermedad incluye la disminución de la productividad (costos indirectos), así como la búsqueda de atención y los gastos médicos directos ${ }^{7}$. Con dichos antecedentes, es necesario priorizar en la búsqueda de nuevas alternativas o estrategias de control y prevención del dengue con miras a disminuir no sólo la carga económica de los países endémicos, sino también a mejorar la calidad de vida de los pacientes. Esto se hace aún más urgente si se tiene en cuenta que aún no se dispone de una vacuna universalmente aprobada, a pesar de las diferentes vacunas candidato evaluadas (Tabla 1). Sin embargo, vale la pena resaltar el enorme progreso alcanzado en dicho campo y actualmente existen varias vacunas que están siendo evaluadas o se tienen en planes para la evaluación ${ }^{8-10}$. El desarrollo de una vacuna contra el dengue se ha visto obstaculizado por muchos desafíos únicos, debido a la existencia de cuatro serotipos del DENV y la inmunidad es serotipo-específica; es decir, la infección con un serotipo determinado confiere inmunidad permanente contra el mismo serotipo (inmunidad homóloga) pero no contra el resto de serotipos, por lo que en teoría, una persona podría padecer dengue hasta cuatro veces a lo largo de su vida (una por cada serotipo). De hecho, es común que individuos desarrollen dengue grave cuando son infectados por segunda vez por un serotipo diferente a la

Tabla 1. Vacunas candidatas actualmente en desarrollo clínico ${ }^{14-16}$

\begin{tabular}{|c|c|c|c|}
\hline Fase I & Fase II & Fase III & $\begin{array}{l}\text { Registrada para } \\
\text { su uso }\end{array}$ \\
\hline $\begin{array}{c}\text { TDENV PIV } \\
\text { (Virus inactivado) }\end{array}$ & $\begin{array}{c}\text { TDV } \\
\text { (Virus atenuado) }\end{array}$ & $\begin{array}{c}\text { TV003 } \\
\text { (Virus atenuado) }\end{array}$ & $\begin{array}{c}\text { CYD-TDV } \\
\text { (Dengvaxia, virus } \\
\text { atenuado) }\end{array}$ \\
\hline \multicolumn{4}{|l|}{$\begin{array}{c}\text { TDENV-LAV + TDEN-PIV } \\
\text { (Virus inactivado y atenuado) }\end{array}$} \\
\hline \multicolumn{4}{|l|}{$\begin{array}{l}\text { TVDV } \\
\text { (DNA plasmídico que codifica } \\
\text { para proteínas de membrana } \\
\text { y envoltura del virus) }\end{array}$} \\
\hline \multicolumn{4}{|l|}{$\begin{array}{c}\text { V180 } \\
\text { (Proteína de membrana y } \\
\text { de envoltura recombinante) }\end{array}$} \\
\hline $\begin{array}{c}\text { TV005 } \\
\text { (Virus atenuado) }\end{array}$ & & & \\
\hline
\end{tabular}

primera infección, debido a la presencia de anticuerpos de reacción cruzada y no neutralizantes que se unen al virus y promueven la infección, en un proceso conocido como potenciación mediada por anticuerpos ${ }^{11,12}$.

Recientemente se han descrito otras estrategias que podrían tener un potencial terapéutico considerable permitiendo sobrepasar muchos de los desafíos que proporciona el DENV. Una de las más prometedoras es el uso de los microARNs (miR). Desde que se describieron y demostró por primera vez que los miR jugaban un papel fundamental en la regulación post-transcripcional de la expresión génica en eucariotes, su aplicación en el campo de la virología ha tenido un continuo crecimiento y se ha sugerido que los miR podrían constituir una nueva estrategia con un gran potencial terapéutico ${ }^{13}$. Los miR son pequeños ARNs no codificantes (21-25 nt) que inhiben o retardan la síntesis de proteínas interactuando con la región 3' no codificante de los miR o ARN virales de polaridad positiva, como el de los flavivirus, entre ellos el DENV. La biogénesis de los miR incluye diferentes etapas ${ }^{18}$. Inicialmente los genes que codifican por los respectivos miRNA son transcriptos por la ARN polimerasa II celular (Figura 1, ruta a), como un pri-miR de más de 200 nucleótidos (nt); luego es procesado en el núcleo por un complejo proteico del que hace parte la enzima conocida como DROSHA, dando origen a un pre-miR de aproximadamente $70 \mathrm{nt}$, constituido por azas y tallos. Los pre-miR son exportados al citoplasma por la exportina 5, donde es nuevamente procesado por DICER, dando origen a un ARN de doble cadena, de aproximadamente $22 \mathrm{nt}$ con un extremo 3'UTR con dos nt de desfase. Dicho ARN es reconocido por un complejo proteico, entre ellas las conocidas como ARGONAUTA; una de las cadenas permanece en el complejo como un miR maduro, formando lo que se conoce como complejo de silenciamiento inducido por ARN (RISC) ${ }^{19}$. Este complejo es el responsable de la degradación del mRNA o de retardar la síntesis de la respectiva proteína. Una de las ventajas de estos miR es que pueden ser generados a partir de vectores (retrovirales) de expresión que producen directamente el pre-miR, lo que constituiría una gran ventaja a la hora de usarse como estrategia terapéutica (Figura 1, ruta b). Otra estrategia utilizada para silenciar ARN mensajeros de interés, es la liberación de los pre-miR, que son muy estables, directamente en la célula mediante liposomas, polímeros o nanopartículas. Sin embargo y a pesar de que este sistema es muy novedoso, se debe ser consciente que aún se requiere de evaluaciones más exhaustivas para que se convierta en una nueva oportunidad terapéutica para el tratamiento de enfermedades de origen viral, como el dengue. Lo interesante de los miR, es que ellos pueden proporcionar el silenciamiento de un gen específico por un tiempo prolongado y así podrían contrarrestar las infecciones virales crónicas, tales como 


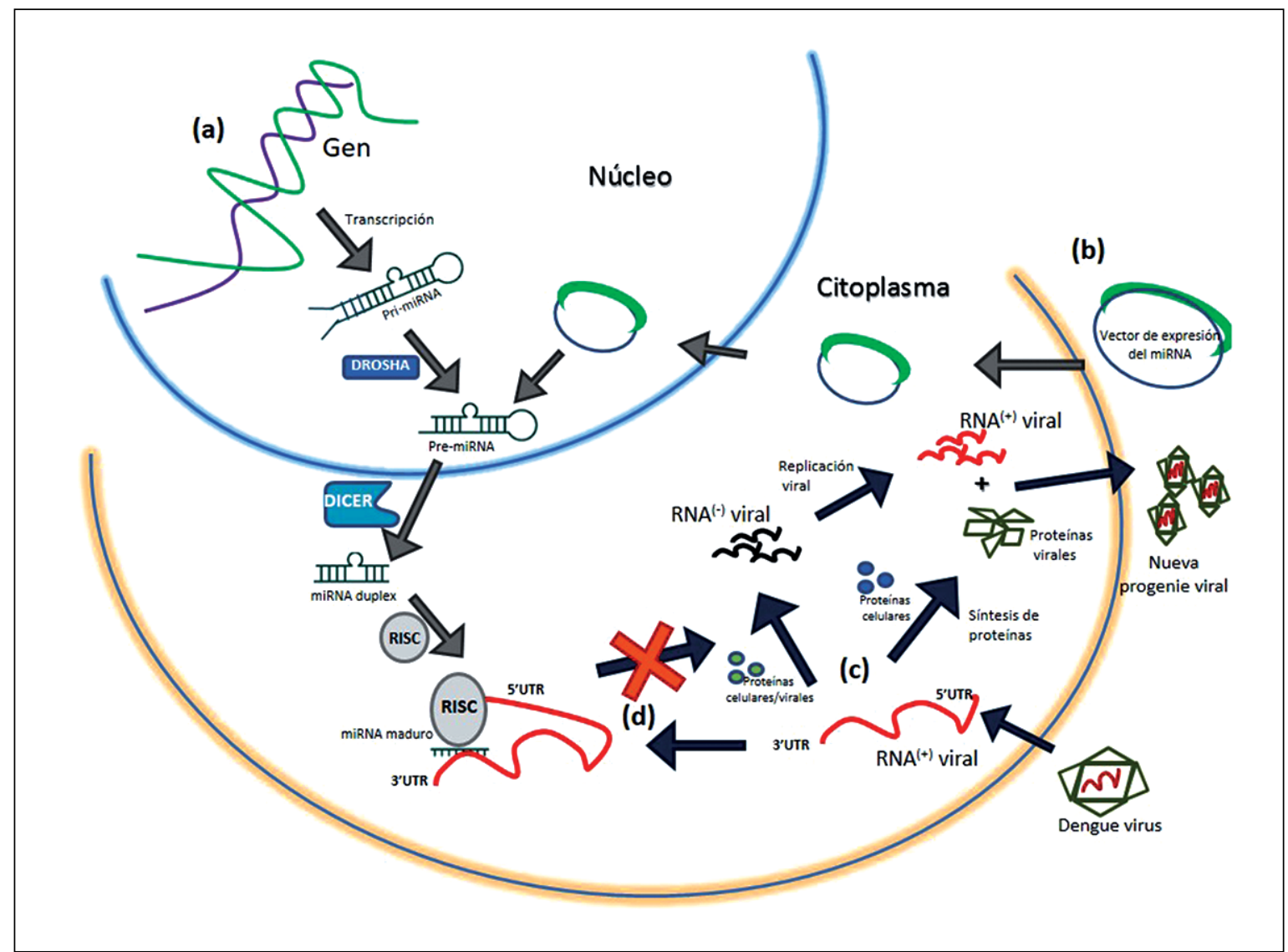

Figura 1. Biogénesis de los miRNA e inhibición de la replicación del DENV. La biogénesis de los miRNA se inicia en el núcleo (a) donde la RNA polimerasa II transcribe el respectivo gen, dando origen a un pri-miRNA. Este es reconocido y procesado por DROSHA (una RNase tipo III) resultando lo que se conoce como pre-miRNA, el cual es exportado al citoplasma por la exportina 5. En el citoplasma es reconocido y clivado por DICER, produciendo el miRNA maduro (22 a $25 \mathrm{nt}$ ) de doble cadena. El miRNA maduro finalmente es "cargado" en el complejo de silenciamiento inducido por RNA (RISC), del cual hacen parte las proteínas conocidas como ARGONAUTA. Ahí, una de las hebras de RNA de degradada y la otra permanece en el complejo, la cual una vez interactúa con el mRNA blanco, o bien lo degrada o retarda la síntesis de la respectiva proteína. Los miRNA también se pueden generar a partir de vectores de expresión (b). En este caso, el gen que codifica por el miRNA de interés se clona en un vector de expresión y se introduce en la célula para así generar el miRNA de interés. También se puede introducir los pre-miRNA directamente en la célula, usando liposomas, polímeros o nanopartículas. En (c) se representa el ciclo de replicación del DENV y en (d) el silenciamiento de la replicación del virus.

las causadas por los virus VIH-1, hepatitis B, hepatitis $\mathrm{C}$ y DENV ${ }^{14,15}$. Para lograr estos objetivos, es necesario identificar los miR celulares capaces de reconocer el ARN viral blanco e inhibir la síntesis de proteínas virales y con ello, la replicación del respectivo virus. O identificar miR implicados en la regulación de la expresión de proteínas implicadas en el ciclo de replicación del virus de interés. Por tanto, el aprovechamiento de esta estrategia debe constituir, dado su potencial, el punto de mira en la lucha contra infecciones virales para las cuales aún no se dispone de estrategias terapéuticas. Por esto, en el Grupo Inmunovirología de la Universidad de Antioquia, una de sus líneas de investigación recientemente enfocó sus estudios hacia la búsqueda de los miR celulares que pudiesen estar implicados en el control de la replicación no sólo de uno, sino de los 4 serotipos del DENV, con el fin de que se pueda desarrollar en un futuro, una terapia basada en la expresión de los miR, para el control de la infección y el tratamiento del dengue.

Básicamente, el objetivo de esta línea de investigación es contribuir a una mejor comprensión de la relación entre los miR celulares y el DENV, de tal manera que nos permite captar el máximo de conocimiento para la mejor comprensión de las funciones biológicas involucradas en dicha interacción. Se debe tener presente que no somos los únicos interesados en este tipo de investigaciones, ya que grandes grupos de investigación alrededor del mundo se han propuesto objetivos similares. Es tanta la 
importancia del tema, que en los últimos 10 años se han publicado más de 25 artículos sobre DENV y miR. Uno de los primeros estudios que demostró una relación entre la infección con flavivirus y los miR fue reportado en mosquitos Aedes y Culex ${ }^{16}$. En el mismo año se reportó que al insertar un elemento de reconocimiento de miR en la región 3' no codificante del ARN del DENV, se alteraba la replicación del virus ${ }^{17}$. Recientemente se reportó que ciertos $\mathrm{miR}$ controlaban la replicación del DENV alterando la expresión de diferentes proteínas implicadas en la respuesta inmune innata u otras vías ${ }^{18,19}$. Pero igualmente se ha descrito que ciertos miR favorecen la replicación del DENV ${ }^{20}$. Actualmente existen muy pocos estudios que evalúen la expresión/regulación de la expresión de miR en pacientes con dengue. Uno de ellos describe el patrón de expresión de miR en sangre de pacientes con dengue y según dicho estudio, $12 \mathrm{miR}$ cambia significativamente el patrón de expresión en pacientes con dengue agudo y $17 \mathrm{miR}$ fueron potencialmente asociados con complicaciones asociadas con dengue ${ }^{21}$. Igualmente se ha reportado cambios muy importantes en la expresión de miR en leucocitos mononucleares de sangre periférica de individuos con dengue ${ }^{22}$. En pacientes con dengue hemorrágico se encontró una disminución significativa en la expresión de SOCS1 e interferón gamma, pero no en pacientes con fiebre por dengue $^{23}$. Además, los investigadores observaron que el incremento en la expresión del miR-150 se asocia con una disminución en la expresión de SOCS1, en monocitos.

Dado que Colombia es un país endémico para el DENV y el dengue tiene un gran impacto tanto a nivel social como económico ${ }^{3}$, nuestro grupo de investigación consciente de dicha problemática, también ha enfocado sus estudios a la búsqueda de alternativas para el control de la infección por el DENV. Gracias a proyectos financiados por Colciencias y el CODI de la Universidad de Antioquia, se ha logrado avances muy importantes relacionados con el papel de los miR en la replicación del DENV. Para ello, lo primero que se hizo fue hacer uso de herramientas bioinformáticas disponibles "online" para identificar posibles miR celulares que reconozcan secuencias blanco en la región 3'UTR del genoma de los cuatro serotipos del DENV. Gracias a esta estrategia se logró identificar $13 \mathrm{miR}$ celulares que cumplían dichos criterios. Lo interesante es que algunos de esos miR tenían como secuencia blanco, regiones del genoma que juegan un papel muy importante ya sea en la circularización del genoma, paso esencial en la síntesis de proteínas y replicación del genoma viral, o en la interacción de factores celulares. Con dicha información, nuestro siguiente paso fue verificar el papel de algunos de esos miR en la replicación del DENV. En particular, nos interesamos por el miR-133a, el miR-744 y el miR-484, los cuales aún no se habían asociado con el DENV. El primero de esos miR es muy interesante ya que está implicado en el control de la expresión de la proteína conocida como PTB (del inglés polypyrimidine tract-binding protein), la cual se ha reportado es esencial para la replicación del $\mathrm{DENV}^{24}$. Nuestros estudios permitieron demostrar efectivamente que la sobre-expresión del miR-133a inhibía la replicación del DENV-2 $2^{25}$. Además pudimos demostrar que la infección por el DENV-2 incrementa la expresión de PTB durante las primeras horas de la infección, pero al mismo tiempo, la presencia del miR-133a contrarrestaba dicho efecto durante las primeras $12 \mathrm{~h}$. Además se pudo observar que el DENV-2 inhibía la expresión endógena del miR-133a durante las primeras $8 \mathrm{~h}$ de la infección. Lo interesante es que este fenómeno se observó para los cuatro serotipos, pero el que suprime más drásticamente la expresión del miR-133a es el DENV-2. Finalmente, demostramos que el DENV controlaba la expresión del miR-133a a través de la región 3'UTR. Esto es muy interesante ya que se ha reportado que la región 3'UTR da origen a lo que se conoce como ARN sub-genómico de flavivirus, el que tiene varias funciones, entre ellas inhibir la respuesta antiviral mediada por lo que se conoce como ARN de interferencia y la producción de interferón ${ }^{26-28}$.

En lo relacionado con los miR-484 y miR-744, igualmente encontramos que inhiben la replicación del DENV. Lo interesante es que en este caso logramos establecer que esos dos miR suprimían la replicación de los cuatro serotipos de DENV ${ }^{29}$. Tal como se observó con el miR-133a, en este estudio también observamos que los cuatro serotipos del DENV inhibían la expresión endógena del miR-484 y miR-744 durante los estadios tempranos de la infección. Dado que nuestros resultados muestran que la región 3'UTR del DENV-1, DENV-2 y DENV-3 suprimen la expresión endógena del miR-484 y miR-744, sugerimos que la región 3'UTR del ARN del DENV interviene activamente en el control de la expresión de los dos miRs objeto de estudio. Teniendo en cuenta nuestros resultados, se propone una nueva estrategia para el control de la replicación del DENV, basada en la sobre-expresión ya sea del miR-133a, del miR-484 o del miR-744. Esta sobre-expresión estaría basada en el uso de vectores de expresión (plásmidos que contienen la secuencia del gen que codifica para un miR específico) que pueden ser fácilmente liberados en las células blanco y expresados establemente por tiempos prolongados (Figura 1b). Igualmente se podrían liberar como pre-miR, usando diferentes estrategias. La ventaja de esta estrategia es que no se tendrían que desarrollar moléculas para cada serotipo del DENV, sino más bien una sola molécula capaz de bloquear la replicación de los cuatro serotipos gracias al reconocimiento de ciertas regiones conservadas presentes en la región 3'UTR de los cuatro serotipos y que juegan un papel fundamental en la replicación viral. 
Sin embargo, somos conscientes que aún hay mucho camino por recorrer y antes de enfocarse en una estrategia terapéutica basada en la sobre-expresión de los miR, es necesario otro tipo de estudios que permitan entender mejor el o los mecanismos de acción de dichos miR implicados en el control de la replicación del DENV. A pesar de ello, consideramos que nuestros hallazgos son un gran aporte para una mejor comprensión de la relación virus-hospedero y un gran avance en la búsqueda de nuevas estrategias que permitan luchar contra el dengue.

En conclusión, los resultados obtenidos con el presente estudio muestran que efectivamente los miR celulares están implicados en el control de la replicación del DENV $\mathrm{y}$, por tanto, su potencial antiviral debe ser considerado en la lucha contra los cuatro serotipos del DENV y así alcanzar el objetivo de reducir la morbilidad y la mortalidad del dengue.

Agradecimientos. A la Universidad de Antioquia.

\section{Resumen}

El dengue es una de las enfermedades más importantes transmitidas por mosquitos y su incidencia ha aumentado a un ritmo alarmante en los últimos años, al punto que se ha convertido en un verdadero problema de salud pública. Actualmente no existe ni vacuna, ni un medicamento o tratamiento adecuado para el control del dengue. Con dichos antecedentes, es necesario priorizar en la búsqueda de nuevas alternativas o estrategias de control y prevención del dengue con miras a disminuir no sólo la carga económica de los países endémicos, sino también a mejorar la calidad de vida de los pacientes. En este sentido, se presenta una breve reflexión sobre algunos aspectos relacionados con la búsqueda de nuevas alternativas en Colombia, enfocadas en el uso de los microARNs, que podrían constituir una nueva estrategia con un gran potencial terapéutico, dado que tendrían el potencial de contrarrestar algunas infecciones virales crónicas.

\section{Referencias bibliográficas}

1.- Bhatt S, Gething P W, Brady O J, Messina J P, Farlow A W, Moyes C L, et al. The global distribution and burden of dengue. Nature 2013; 496: 504-7.

2.- Guzmán M G, Kourí G. Dengue diagnosis, advances and challenges. Int Infect Dis 2004; 8: 69-80.

3.- Castrillón J C, Carlos J, Urcuqui S. Dengue en Colombia, diez años de evolución. Rev Chilena Infectol 2015; 32: 142-9.

4.- Fares R C G, Souza K P R, Añez G, Ríos M. Epidemiological scenario of Dengue in Brazil. Bio Med Res Int 2015; 2015: 1-13.

5.- OMS 2009. Dengue Guias para el Diagnóstico, Tratamiento, Prevencion y Control. OMS y Programa Espec Para Investig y Capacit Enfermedades Trop 2009; 113-5.

6.- WHO. Dengue and severe dengue 2016. Citado el 14 de marzo de 2016. Disponible en: http:// www.who.int/mediacentre/factsheets/fs117/en/.

7.- Shepard D S, Coudeville L, Halasa Y A, Zambrano B, Dayan G H. Economic impact of dengue illness in the Americas. Am J Trop Med Hyg 2011; 84: 200-7.

8.- Ramakrishnan L, Radhakrishna Pillai M, Nair R R. Dengue vaccine development: strategies and challenges. Viral Immunol 2015; 28: 76-84.

9.- Satterfield B A, Dawes B E, Milligan G N. Status of vaccine research and development of vaccines for Nipah virus. Vaccine 2016; 34 : 2971-5.
10.- Martin J, Hermida L. Dengue vaccine: an update on recombinant subunit strategies. Acta Virol 2016; 60: 3-14.

11.- Welsh R M, Che J W, Brehm M A, Selin L K. Heterologous immunity between viruses. Immunol Rev 2010; 235: 244-66.

12.- Castro-Mussot M E, Machain-Williams C, Lorono-Pino M A, Salazar M I. Respuesta inmune e inmunopatogenesis en las infecciones con el virus del dengue. Gac Med Mex 2013; 149: 531-40.

13.- Louten J, Beach M, Palermino K, Weeks M, Holenstein G. MicroRNAs Expressed during viral infection: biomarker potential and therapeutic Considerations. Biomark Insights 2015; 10: 25-52.

14.- Skalsky R L, Cullen B R. Viruses and microRNAs. Reference Module in Biomedical Research 2014.

15.- Arbuthnot P. MicroRNA-like antivirals. Biochim Biophys Acta-Gene Regul Mech 2011; 1809: 746-55.

16.- Skalsky R L, Vanlandingham D L, Scholle F, Higgs S, Cullen B R. Identification of microRNAs expressed in two mosquito vectors, Aedes albopictus and Culex quinquefasciatus. BMC Genomics 2010; 11: 1-16.

17.- Lee T C, Lin Y L, Liao J T, Su C M, Lin C C, Lin W P, et al. Utilizing liver-specific microRNA-122 to modulate replication of dengue virus replicon. Biochem Biophys Res Commun 2010; 396: 596-601.

18.- Wu N, Gao N, Fan D, Wei J, Zhang J, An J. miR-223 inhibits dengue virus replication by negatively regulating the microtubuledestabilizing protein STMN1 in EAhy926 cells. Microbes Infect 2014; 15: 911-22.

19.- Zhu X, He Z, Hu Y, Wen W, Lin C, Yu J, et al. MicroRNA-30e* suppresses dengue virus replication by promoting NF-kappaBdependent IFN production. PLoS Negl Trop Dis 2014; 8: e3088.

20.- Henke J I, Goergen D, Zheng J, Song Y, Schuttler C G, Fehr C, et al. microRNA-122 stimulates translation of hepatitis $\mathrm{C}$ virus RNA. EMBO J 2008; 27: 3300-10.

21.- Tambyah P A, Ching C S, Sepramaniam S, Ali J M, Armugam A, Jeyaseelan K. microRNA expression in blood of dengue patients. Ann Clin Biochem 2016; 53: 466-76.

22.- Qi Y, Li Y, Zhang L, Huang J. MicroRNA expression profiling and bioinformatic analysis of dengue virus-infected peripheral blood mononuclear cells. Mol Med Rep 2013; 7: 791-8.

23.- Chen R F, Yang K D, Lee I K, Liu J W, Huang $\mathrm{C} \mathrm{H}$, Lin C Y, et al. Augmented miR-150 expression associated with depressed SOCS1 expression involved in dengue haemorrhagic fever. J Infect 2014; 69: 366-74.

24.- De Nova-Ocampo M, Villegas-Sepúlveda N, del Angel R M. Translation elongation factor1alpha, La, and PTB interact with the 3' untranslated region of dengue 4 virus RNA. Virology 2002; 295: 337-47.

25.- Castillo J A, Castrillón J C, Diosa-Toro M, Betancur J G, III GSL, Smit J M, et al. Complex interaction between dengue virus 
replication and expression of miRNA-133a. BMC Infect Dis 2016; 16: 1-12.

26.- Schnettler E, Sterken M G, Leung J Y, Metz S W, Geertsema C, Goldbach R W, et al. Noncoding flavivirus RNA displays RNA interference suppressor activity in insect and mammalian cells. J Virol 2012; 86: 13486-500.
27.- Moon S L, Dodd B J T, Brackney D E, Wilusz C J, Ebel G D, Wilusz J. Flavivirus sfRNA suppresses antiviral RNA interference in cultured cells and mosquitoes and directly interacts with the RNAi machinery. Virology 2015; 485: 322-9.

28.- Manokaran G, Finol E, Wang C, Gunaratne J, Bahl J, Ong E Z, et al. Dengue subgenomic
RNA binds TRIM25 to inhibit interferon expression for epidemiological fitness. Science 2015; 350: 217-21.

29.- Castrillón-Betancur J C, Urcuqui-Inchima S. Overexpression of miR-484 and miR-744 in Vero cells alters replication of Dengue virus. Mem Inst Oswaldo Cruz, Río de Janeiro 2017: 1-11 doi: 10.1590/0074-02760160404.0 